COMUNICAÇÃO CIENTÍFICA

\title{
ESCURECIMENTO INTERNO DE PÊRA ASIÁTICA 'YA LI' DURANTE O ARMAZENAMENTO REFRIGERADO'
}

\author{
AURI BRACKMANN² ${ }^{2}$ MARCELO CERETTA ${ }^{3}$, MAURÍCIO HUNSCHE $^{4}$, ALINE FRANKEN $^{5}$
}

\begin{abstract}
RESUMO - A pêra 'Ya Li' apresenta escurecimento da polpa poucos dias após o armazenamento refrigerado. Este trabalho objetivou avaliar condições de armazenamento visando a diminuir os danos pela baixa temperatura inicial em peras ' $\mathrm{Ya}$ Li'. As condições iniciais estabelecidas foram: 1) $0^{\circ} \mathrm{C}$;2) $5^{\circ} \mathrm{C}$, nos 10 primeiros dias, com redução de $0,7^{\circ} \mathrm{C}$ por dia, durante sete dias; 3) $10^{\circ} \mathrm{C}$ nos 10 primeiros dias, com redução de $1,4^{\circ} \mathrm{C}$ por dia, durante sete dias; 4$) 10^{\circ} \mathrm{C}$ nos 10 primeiros dias, com redução de $1,4^{\circ} \mathrm{C}$ por dia, durante sete dias, sempre em atmosfera controlada $\left(2,0 \mathrm{kPa}\right.$ de $\mathrm{O}_{2} \mathrm{e}<1,0 \mathrm{kPa}$ de $\left.\left.\mathrm{CO}_{2}\right) ; 5\right) 10^{\circ} \mathrm{C}$ nos 10 dias iniciais, com posterior exposição direta dos frutos a $0^{\circ} \mathrm{C}$. Em seqüência aos tratamentos iniciais, os frutos foram submetidos ao armazenamento refrigerado a $0^{\circ} \mathrm{C}$, com exceção do tratamento " 4 " que foi mantido continuamente em atmosfera controlada. Após 52 dias de armazenamento, verificou-se escurecimento interno, superior a $68 \%$ em todos os tratamentos. As temperaturas mais elevadas, no início, não evitaram a ocorrência do escurecimento durante o armazenamento.
\end{abstract}

Termos para indexação: Pyrus bretschneideri, pós-colheita, degenerescência, escurecimento interno.

\section{INTERNAL BROWNING OF 'YA LI' ASIAN PEARS DURING COLD STORAGE}

\begin{abstract}
Ya Li" pears shows internal browning soon after a few storage days. This work evaluated storage conditions aiming to reduce low temperature injury. The established conditions were: 1) $0^{\circ} \mathrm{C}$;2) $5^{\circ} \mathrm{C}$ on the 10 first days, reducing $0.7^{\circ} \mathrm{C}$ per day during 7 days; 3) $10^{\circ} \mathrm{C}$ on the 10 first days, reducing $1,4^{\circ} \mathrm{C}$ per day during 7 days; 4) $10^{\circ} \mathrm{C}$ on the 10 first days, reducing $1.4^{\circ} \mathrm{C}$ per day during 7 days, always under controlled atmosphere $\left(2.0 \mathrm{kPa} \mathrm{O}_{2}\right.$ and $\left.\left.<1.0 \mathrm{kPa} \mathrm{CO}_{2}\right) ; 5\right) 10^{\circ} \mathrm{C}$ on the 10 first days with posterior exposure to $0^{\circ} \mathrm{C}$. After the initial treatments, fruits were submitted to cold storage at $0^{\circ} \mathrm{C}$, with exception of treatment " 4 " stored continuosly under controlled atmosphere. After 52 storage days, internal browning was higher than $68 \%$ in all treatments. Higher temperatures at the beginning of the storage did not avoid the internal browning during storage.
\end{abstract}

Index terms: Pyrus bretschneideri, postharvest, flesh breakdown, internal browning.

A pêra asiática tem tido um aumento de produção e uma boa aceitação pelos consumidores brasileiros, devido às suas qualidades organolépticas. Entretanto, produtores do Sul do Brasil vêm enfrentando significativas perdas com a cultivar $\mathrm{Ya} \mathrm{Li}$ em função do seu rápido escurecimento interno durante armazenamento refrigerado. Este distúrbio fisiológico pode estar relacionado a vários fatores, tais como época de colheita (Crisosto et al., 1994), temperatura inicial de armazenamento (Ju et al., 1994) ou concentrações de gases, principalmente o $\mathrm{CO}_{2}$ (Zagori et al., 1989). As peras sofrem influências consideráveis em seu armazenamento com a variação da temperatura. Em trabalhos desenvolvidos por Zhou (1992), Chen et al. (1992) e Ju et al. (1994), constatou-se que a temperatura ótima para armazenamento da cultivar Ya Li situa-se em $0^{\circ} \mathrm{C}$. Entretanto, baixas temperaturas no início do armazenamento parecem ser responsáveis pelo aparecimento de distúrbios fisiológicos. Esses autores sugerem, para diminuir o escurecimento interno, um armazenamento inicial com temperatura ao redor de 12 a $13^{\circ} \mathrm{C}$, com subseqüente redução gradual desta, até atingir $0^{\circ} \mathrm{C}$.

Portanto, devido à inexistência de informações sobre condições de armazenamento de pêra asiática cv. Ya Li produzida no Brasil, desenvolveu-se um experimento com o objetivo de testar condições iniciais de armazenamento que evitem o posterior escurecimento interno dos frutos durante o armazenamento. Os frutos foram colhidos em 3 de março de 2000, provenientes de um pomar comercial do município de Castro-PR. Divididos em cinco tratamentos, utilizou-se um delineamento experimental inteiramente casualizado, com quatro repetições e unidade experimental de 18 frutos. Os tratamentos foram: 1) $0^{\circ} \mathrm{C}$;2) $5^{\circ} \mathrm{C}$, nos 10 primeiros dias, com redução gradual de $0,7^{\circ} \mathrm{C}$ por dia; 3 ) $10^{\circ} \mathrm{C}$ nos primeiros 10 dias, com redução gradual de $1,4^{\circ} \mathrm{C}$ por dia, durante sete dias; 4) $10^{\circ} \mathrm{C}$ nos primeiros 10 dias, com redução de $1,4^{\circ} \mathrm{C}$ por dia, durante sete dias, sendo este tratamento mantido sempre em atmosfera controlada, 2,0kPa de $\mathrm{O}_{2} \mathrm{e}<1,0 \mathrm{kPa}$ de $\mathrm{CO}_{2}$; 5) $10^{\circ} \mathrm{C}$ nos primeiros 10 dias e, após este período, submetido a $0^{\circ} \mathrm{C}$. Após os tratamentos, todos os frutos foram mantidos em

1 (Trabalho 065/2001). Recebido: 14/03/2001. Aceito para publicação: 13/01/2002.

2 Engenheiro Agrônomo, Professor, Doutor do Departamento de Fitotecnia da Universidade Federal de Santa Maria (UFSM), 97105-900, RS. Autor para correspondência. brackman@creta.ccr.ufsm.br

3 Engenheiro Agrônomo, Mestre, aluno do Programa de Pós-Graduação em Agronomia da Universidade Federal de Pelotas. Bolsista do CNPq.

4 Engenheiro Agrônomo, Mestre em Agronomia - UFSM.

5 Acadêmico do curso de Agronomia, bolsista da SESUL/PET. 


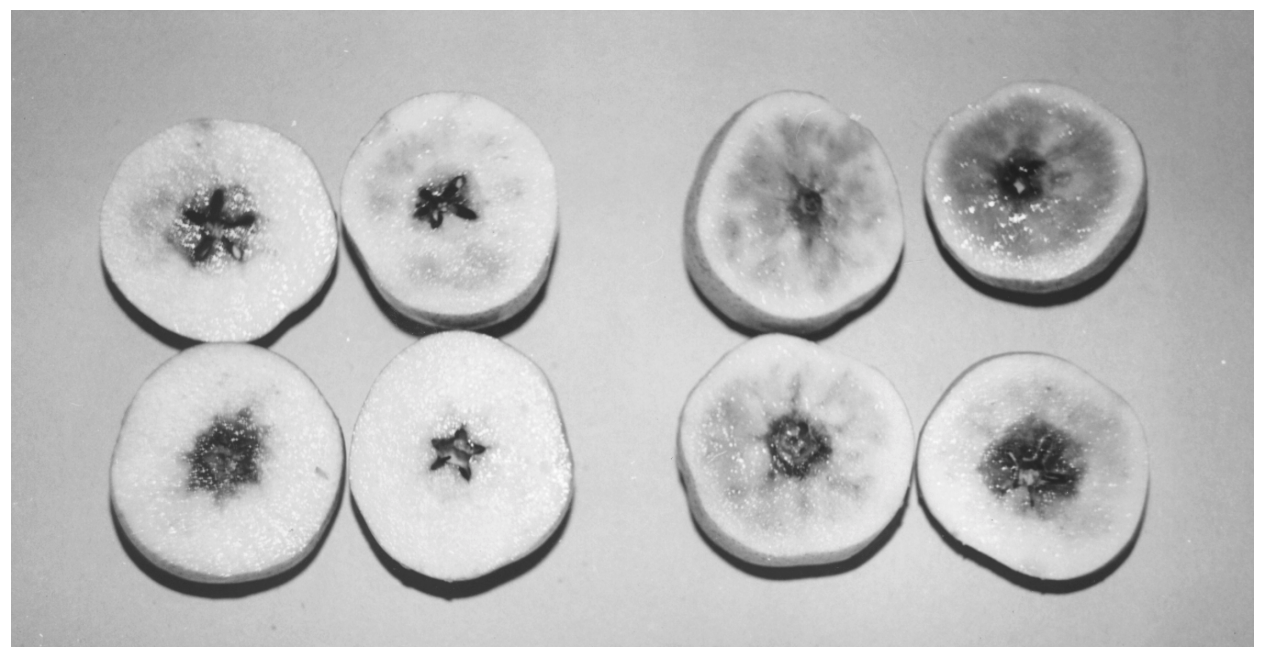

FIGURA 1 - Escurecimento interno em pêra 'Ya Li'. Estágio inicial na região do miolo (Esquerda) e mais evoluído, na polpa (Direita).

TABELA 1 - Percentagem de escurecimento interno em pêra cv. Ya Li, após 52 dias de armazenamento em diferentes regimes.

\begin{tabular}{|c|c|}
\hline Condição inicial de armazenamento & Escurecimento interno $(\%)$ \\
\hline $0^{\circ} \mathrm{C}$ & $68,2 \quad \mathrm{c} * *$ \\
\hline $5^{\circ} \mathrm{C}$ por 10 dias $+\mathrm{RGT}^{*}\left(0,7^{\circ} \mathrm{C} / \mathrm{dia} \times 7\right.$ dias $)$ & $95,3 \mathrm{ab}$ \\
\hline $10^{\circ} \mathrm{C}$ por 10 dias $+\operatorname{RGT}\left(1,4^{\circ} \mathrm{C} /\right.$ dia $\times 7$ dias $)$ & $100 \mathrm{a}$ \\
\hline $10^{\circ} \mathrm{C}$ por 10 dias $+\operatorname{RGT}\left(1,4^{\circ} \mathrm{C} /\right.$ dia $\times 7$ dias $)+2 \mathrm{kPa}$ de $\mathrm{O}_{2} \mathrm{e}<1 \mathrm{kPa}$ de $\mathrm{CO}_{2}$ & $100 \mathrm{a}$ \\
\hline $10^{\circ} \mathrm{C}$ por 10 dias & $88,1 \mathrm{~b}$ \\
\hline C.V $(\%)$ & 6,75 \\
\hline
\end{tabular}

* RGT $=$ Redução Gradual de Temperatura

** Médias não seguidas pela mesma letra diferem entre si, pelo teste de Duncan, a 5\%.

armazenamento refrigerado (AR), a $0^{\circ} \mathrm{C}$, com umidade relativa (UR) de $95 \pm 1 \%$, com exceção do tratamento em $\mathrm{AC}$, que foi mantido nas mesmas condições iniciais de $\mathrm{O}_{2}$ e $\mathrm{CO}_{2}$ e UR de $97 \pm$ $1 \%$. Aos 52 dias de armazenamento, a $0^{\circ} \mathrm{C}$, avaliou-se visualmente o escurecimento da polpa dos frutos, através de diversos cortes transversais, sendo os resultados expressos em percentagem.

Todos os tratamentos apresentaram valores elevados de escurecimento interno (Tabela 1). Entretanto, o armazenamento imediato a $0^{\circ} \mathrm{C}$, desde o primeiro dia, foi a condição que apresentou menor incidência desse distúrbio. Estes dados afastam a possibilidade de esta temperatura, desde o início do armazenamento, ser a causa primária do escurecimento. Porém Zhou (1992) e Chen et al. (1992), trabalhando com esta mesma cultivar, relataram maior escurecimento interno com o armazenamento contínuo a $0^{\circ} \mathrm{C}$. Contrariamente ao esperado, as temperaturas de 5 e $10^{\circ} \mathrm{C}$, no início, apresentaram percentual de escurecimento que variou de 88,1 a $100 \%$, sendo mais elevado do que a $0^{\circ} \mathrm{C}$, não tendo controlado este distúrbio fisiológico. Resultados diferentes foram obtidos por Zhou (1992), e Chen et al. (1992), que verificaram menor escurecimento quando os frutos foram previamente armazenados a 13 e $12^{\circ} \mathrm{C}$, respectivamente. $\mathrm{O}$ escurecimento caracterizou-se por iniciar pela região do miolo e, posteriormente, progredir para a polpa (Figura 1). A causa desta desordem em peras asiáticas ainda é desconhecida (Crisosto et al., 1994b). Estes mesmos autores afirmam que a 'Ya Li', quando colhida precocemente, mas em estágio comercial, não apresentou nenhum escurecimento a $0^{\circ} \mathrm{C}, 10^{\circ} \mathrm{C}$ ou $20^{\circ} \mathrm{C}$, porém, quando colhida mais tardiamente, o escurecimento foi próximo a $100 \%$, dependendo, portanto, da data de colheita e não da temperatura de armazenamento.

Conclui-se, portanto, que o armazenamento inicial a temperaturas de $0^{\circ} \mathrm{C}, 5^{\circ} \mathrm{Ce} 10^{\circ} \mathrm{C}$, mesmo em atmosfera controlada, não é apropriado para o controle do escurecimento interno de pêra 'Ya Li', sugerindo-se novos trabalhos de pesquisa com diferentes pontos de maturação para as peras produzidas nas condições brasileiras.

\section{REFERÊNCIAS BIBLIOGRÁFICAS}

CHEN, K.S., JU, L., ZHOU, S.T. Comparison of fruit storage behaviour in the pear cultivars Yali and Xuehua. Plant Physiology Communications, Pequin, v.28, n.6, p.428-430, 1992.

CRISOSTO, C.H., GARNER, D., CRISOSTO, G.M., et al. Late harvest and delayed cooling induce internal browning of ' $\mathrm{Ya} \mathrm{Li}$ ' and 'Seuri' chinese pears. HortScience, Alexandria, v.29, n.6, p.667-670, 1994.

CRISOSTO, C.H., GARNER, D., CRISOSTO, G.M Early harvest prevents internal browning in Asian Pears. California 
Agriculture, California, v.48, n.4, p.17-19, 1994 b.

JU,Z.G., YUAN, Y.B., LIOU, C.L., et al. Effects of low temperature on $\mathrm{H}_{2} \mathrm{O}_{2}$ and heart browning of Chili and Yali (Pyrus bretschneideri R.). Scientia Agricultura Sinica, Pequin, v.27, n.5, p.77-81, 1994.

ZAGORY, D., KE, D., KADER, A.A. Long term storage of Early gold and 'Shinko' asian pear in low oxygen atmosphere. In: INT. CONTR. ATM. RES. CONF., 8., 1989, Washington. Proceedings..., v.1, p.353-557.

ZHOU, H.W. Effect of chilling rate on physiology of Yali pear in storage. Journal of Fruit Science, Pequin, v.9, n.1, p.36-38, 1992. 